\title{
Stem cell fate determination during development and regeneration of ectodermal organs
}

\section{Lucía Jiménez-Rojo*, Zoraide Granchi, Daniel Graf and Thimios A. Mitsiadis}

Institute of Oral Biology, Zentrum für Zahnmedizin, Faculty of Medicine, University of Zurich, Zurich, Switzerland

Edited by:

Anis Feki, Geneva University

Hospitals, Switzerland

\section{Reviewed by:}

Francisco H. Andrade, University of Kentucky College of Medicine, USA Petros Papagerakis, University of Michigan, USA

${ }^{*}$ Correspondence:

Lucía Jiménez-Rojo, Institute of Oral Biology, Faculty of Medicine,

University of Zurich, Plattenstrasse

11, 8032 Zurich, Switzerland.

e-mail: lucia@jimenez-rojo.com
The development of ectoderm-derived appendages results in a large variety of highly specialized organs such as hair follicles, mammary glands, salivary glands, and teeth. Despite varying in number, shape, and function, all these ectodermal organs develop through continuous and reciprocal epithelial-mesenchymal interactions, sharing common morphological and molecular features especially during their embryonic development. Diseases such as ectodermal dysplasias can affect simultaneously these organs, suggesting that they may arise from common multipotent precursors residing in the embryonic ectoderm. During embryogenesis, these putative ectodermal stem cells may adopt different fates and consequently be able to generate a variety of tissue-specific stem cells, which are the sources for the various cell lineages that form the diverse organs. The specification of those common epithelial precursors, as well as their further lineage commitment to tissue-specific stem cells, might be controlled by specific signals. It has been well documented that Notch, Wnt, bone morphogenetic protein, and fibroblast growth factor signaling pathways regulate cell fate decisions during the various stages of ectodermal organ development. However, the in vivo spatial and temporal dynamics of these signaling pathways are not yet well understood. Improving the current knowledge on the mechanisms involved in stem cell fate determination during organogenesis and homeostasis of ectodermal organs is crucial to develop effective stem cell-based therapies in order to regenerate or replace pathological and damaged tissues.

\section{DEVELOPMENT OF ECTODERMAL TISSUES AND THEIR APPENDAGES}

During early embryogenesis, the ectoderm represents the external germ layer, which is composed of the surface ectoderm, neural crest, and neural tube. From the surface ectoderm the epidermis (i.e., skin) and other stratified epithelia (e.g., oral epithelium; Carlson, 2004) develop. Subsequently, these tissues give rise to diverse specialized structures (called ectodermal appendages) such as hair follicles, mammary glands, salivary glands and teeth.

All ectodermal appendages develop through similar cellular mechanisms that involve an intimate and controlled crosstalk between the epithelium and mesenchyme, and share common morphological features during early organogenesis (Pispa and Thesleff, 2003). The first morphological sign of an appendage development is a thickening of the epithelium that forms the appendage placode. In most of the cases the placode invaginates into the mesenchyme and due to an important proliferation activity gives rise to a bud, while the surrounding mesenchyme starts to condense. The bud epithelium grows further into the adjacent mesenchyme and finally acquires an organ-specific configuration (Figure 1).

\section{SPECIFICATION OF ECTODERMAL APPENDAGES: THE ROLE OF EPITHELIAL-MESENCHYMAL INTERACTIONS}

The precise mechanisms that define the specificity of the ectodermal appendages along the body are not yet well understood.
Tissue recombination assays are valuable for investigating the role of the epithelium and mesenchyme in regulating the initiation and morphogenesis of ectoderm-derived organs.

Classical heterotopic tissue recombination experiments, in which epithelium and mesenchyme from different origin are reassociated, have demonstrated the importance of the mesenchyme for initiating the development, and establishing the pattern, of ectodermal organs (Kollar and Baird, 1969; Kratochwil, 1969; Sakakura et al., 1976; Sengel, 1990). The capacity of the mesenchyme to ectopically induce the formation of ectodermal organs has been shown in a large variety of experimental models. For instance, it has been demonstrated that the embryonic mammary mesenchyme has the ability to induce the formation of mammary buds in areas where mammary glands normally do not develop, such as in mid-ventral and dorsal epidermal regions (Cunha et al., 1995). Similarly, it has been established that embryonic dental mesenchyme is able to induce the formation of dental structures when combined with non-dental epithelia in vitro (Mina and Kollar, 1987). Likewise, ex vivo experiments have shown that the oral epithelium of birds is capable of forming tooth-like structures when in contact with mouse neural crest-derived mesenchyme (Mitsiadis et al., 2003, 2006). It is now broadly accepted that the mesenchyme regulates the morphogenetic events of the various ectodermal organs. However, it remains controversial whether the cytodifferentiation events occurring later in the epithelium are tissue-autonomous or strictly mesenchyme-dependent. For 
example, the recombination of mammary epithelium with salivary gland mesenchyme gives rise to salivary glands (Figure 2) that secrete milk proteins instead of salivary proteins (Sakakura et al., 1976). In contrast to the previous findings, embryonic chick mesenchyme (dermis) has been shown to control the epithelial (epidermal) cell differentiation (Dhouailly et al., 1978): tarsometatarsal scale-forming dermis instructs the dorsal epidermis to develop scales, while dorsal feather-forming dermis induces the formation of feathers in tarso-metatarsal epidermis. One possible explanation is that mammary epithelial cells may be committed to express tissue-specific proteins earlier than feather and scale epidermal cells. Along these lines, tissue recombination experiments can provide valuable information on the developmental stage in which tissue-specific stem cells are irreversibly committed. This has been elegantly demonstrated by heterochronal recombination assays using pituitary epithelium and salivary gland mesenchyme

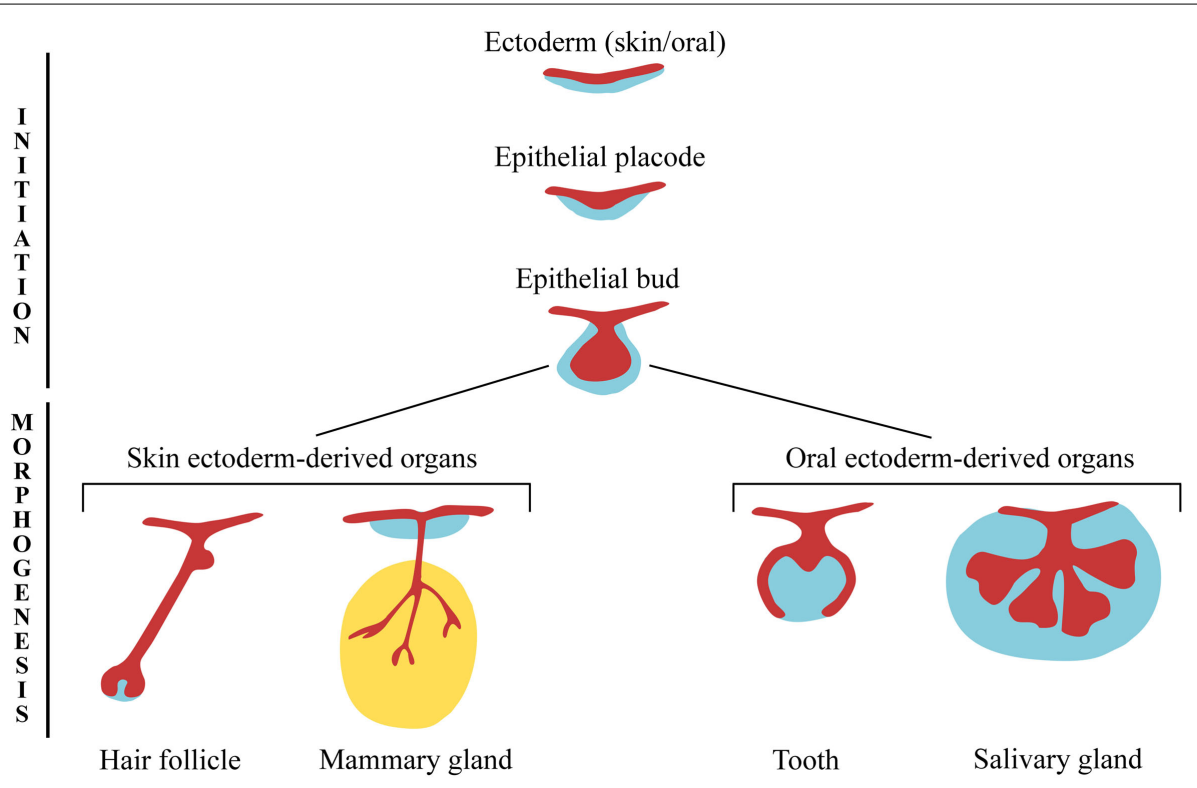

FIGURE 1 | Ectodermal appendages during early stages of development. Ectoderm-derived structures start to develop from embryonic ectoderm upon mesenchymal inductive signals. The formation of the epithelial placodes, and their subsequent growth into the mesenchyme, is common to the early development of all ectodermal organs. At later developmental stages, epithelial buds undergo different morphogenetic programs resulting in the formation of highly specialized structures.

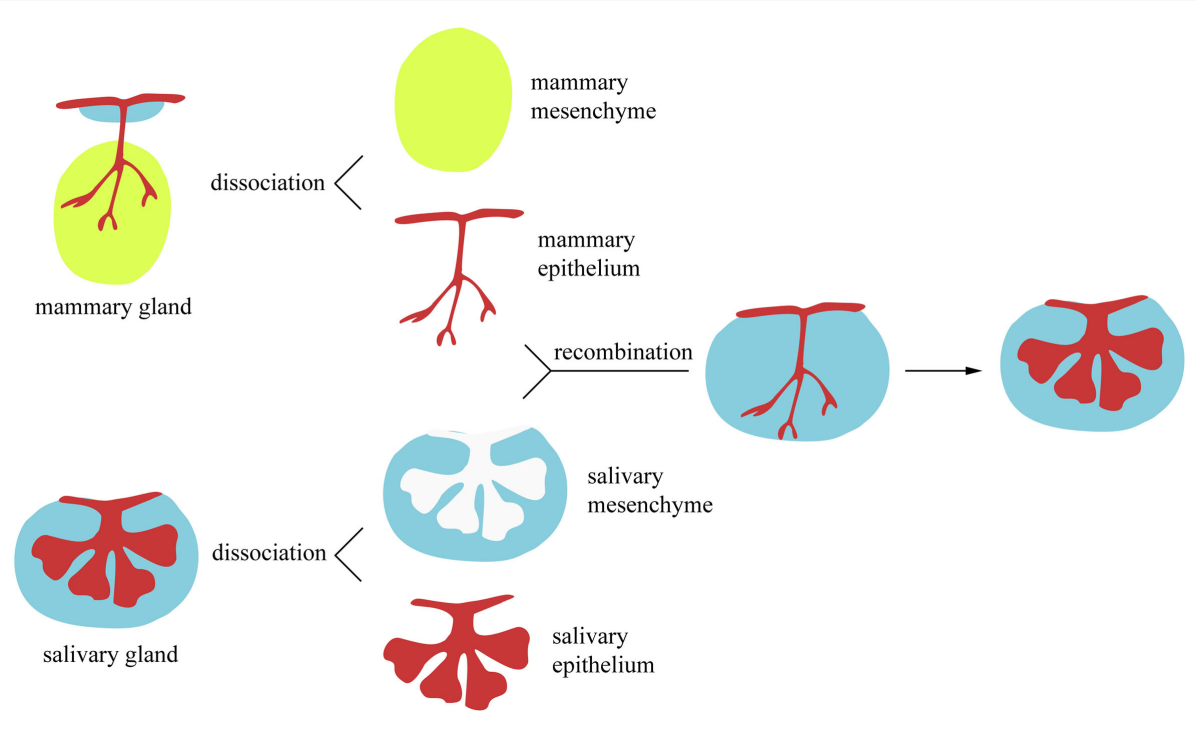

FIGURE 2 | In vitro heterotopic tissue recombination assay. Epithelial and mesenchymal components from ectodermal appendages can be enzymatically and mechanically dissociated. Subsequently, they can be recombined with epithelium and mesenchyme from a different organ. 


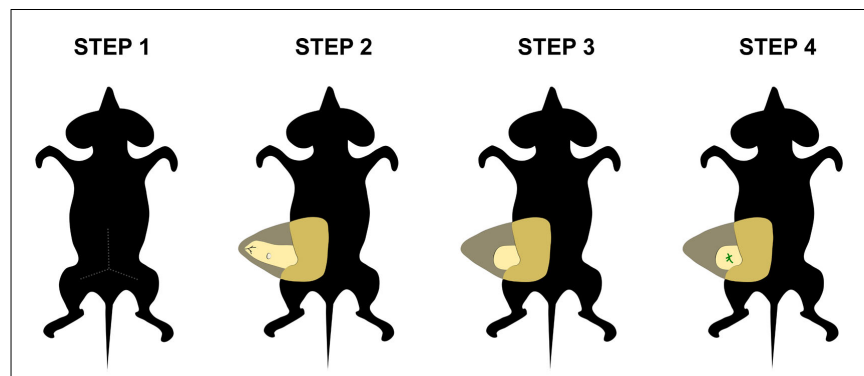

FIGURE 3 | Transplantation into postnatal mammary fat pad. This in vivo tissue recombination assay can be used to assess the effect of the adult mammary mesenchyme in the fate determination of stem cells. First, an incision is made in the abdominal skin of the host mouse (step 1) and the skin is pulled away from peritoneal wall to expose the mammary fat pad (step 2). Subsequently, the fat pad tissue from the lymph node to the nipple area is removed (step 3). Finally, donor tissue is transplanted into the cleared fat pad (step 4).

(Kusakabe et al., 1985). Recombination of embryonic day 8 (E8) to E11 pituitary epithelia with salivary gland mesenchyme results in the formation of salivary epithelial structures. However, this was not the case when pituitary epithelium from more advanced developmental stages was used.

Although it has been well established that the mesenchyme or environment plays an essential role in the epithelial cell fate determination in several ectodermal organs, this may vary by tissue and developmental time point. Does the mesenchyme retain its inductive capacity after birth? Is the microenvironment able to redirect the fate of stem cells? The mouse mammary gland constitutes an ideal model to address and clarify these questions in vivo, because epithelium can be easily removed from the fourth inguinal mammary gland of pre-pubertal mice (Figure 3). Thus, adult and embryonic mammary epithelium can reconstitute a functional mammary gland when transplanted into a cleared or epithelium-free mammary fat pad (Smith and Medina, 1988). By contrast, epithelia from another origin such as salivary gland, lung, and pancreas, fail to undergo morphogenesis when transplanted into the mammary fat pad of postnatal mice (Sakakura et al., 1987). However, it has been demonstrated that postnatal mammary mesenchyme has the potential to induce the differentiation of non-mammary cells into milk-secreting luminal cells after mixing with mammary epithelial cells and subsequently injecting them into empty fat pads (Boulanger et al., 2007, 2012; Booth et al., 2008). Based on their common origin, it would be interesting to study the morphogenetic and differentiation potential of stem cells from various ectodermal-derived organs after their transplantation into mammary mesenchyme, either alone or in combination with mammary epithelial cells.

\section{LINEAGE COMMITMENT OF STEM CELLS DURING DEVELOPMENT OF ECTODERMAL ORGANS}

As mentioned above, skin and oral epithelia are derivatives from the embryonic surface ectoderm. Thus, during embryogenesis, a putative common stem cell population residing in the surface ectoderm would be determined to give rise to multipotent stem cells in skin and oral epithelia that would be further specialized to form ectodermal appendages (Figure 4). For instance, during mammalian embryogenesis, stem cells from the oral epithelium are committed to form a stratified oral mucosa, the highly specialized dental epithelium and salivary and pituitary gland epithelia, whereas stem cells from the skin ectoderm can give rise to epidermis, hair follicles as well as sebaceous, and mammary glands.

Most of the ectodermal organs undergo their terminal differentiation and become functional at postnatal stages. This holds true for salivary glands that complete their functional differentiation during postnatal stages. Similarly, mammary glands reach their major specialization during adulthood and notably during pregnancy. Finally, hair follicles fully develop and cycle only postnatally and teeth erupt after birth. However, the main morphogenetic processes during the development of ectodermal-derived organs occur during embryonic stages. During the morphogenetic stage, tissue-specific stem cells divide and give rise to more committed progenitors that will eventually differentiate into all the tissue-specific lineages (Figure 4).

In the hair follicle, eight different cell types originate from the epithelium. The cells directly responsible for the hair shaft (medulla, cortex, cuticle) formation; the IRS (Inner Root Sheath) lineages (cuticle, Huxley's layer, Henle's layer, and companion layer), which constitute the external support to the shaft; the outer root sheath (ORS) linage, which separates the hair follicle-organ from the epidermis (Niemann and Watt, 2002).

In the adult mammary epithelium, two main cell lineages can be found: the contractile myoepithelial cells and the luminal cells that can be further subdivide into the ductal and the milk-producing alveolar lineages (Woodward et al., 2005).

The salivary gland is composed of acinar, ductal, and myoepithelial cells. Acinar cells can be subdivided into serous or mucous, depending on the composition of their secretion. Similarly, ductal cells can be grouped into three different lineages based on the duct type in which they reside, i.e., intercalated, striated, or granular ductal cells (Tucker, 2007).

Regarding the tooth, epithelium can give rise to outer enamel epithelium, stellate reticulum, stratum intermedium, and inner enamel epithelium (Figure 5) that eventually will differentiate into enamel-secreting ameloblasts (Mitsiadis and Graf, 2009). Lineage tracing experiments using lipophilic carbocyanine dyes, such as the fluorescent dye DiI, have contributed to the understanding of lineage commitment during odontogenesis (Figure 5). In order to identify putative multipotent stem cells in the epithelium of embryonic mouse molars, cells from different regions of the dental epithelium were labeled before the morphogenesis stage. At further stages, DiI dye is retained in the same area where it was injected, suggesting that the labeled cells were committed progenitors rather than multipotent stem cells (Mitsiadis et al., 2008). However, DiI labeling faces several limitations such as the dilution of the dye concentration after each cell division, and it cannot be ruled out the possibility of the existence of multipotent epithelial stem cells in the embryonic molars that were not targeted with DiI.

\section{ADULT EPITHELIAL STEM CELLS IN ECTODERMAL ORGANS}

There is increasing evidence for the existence of a pool of adult stem cells in several tissues that serves as a source for tissue 


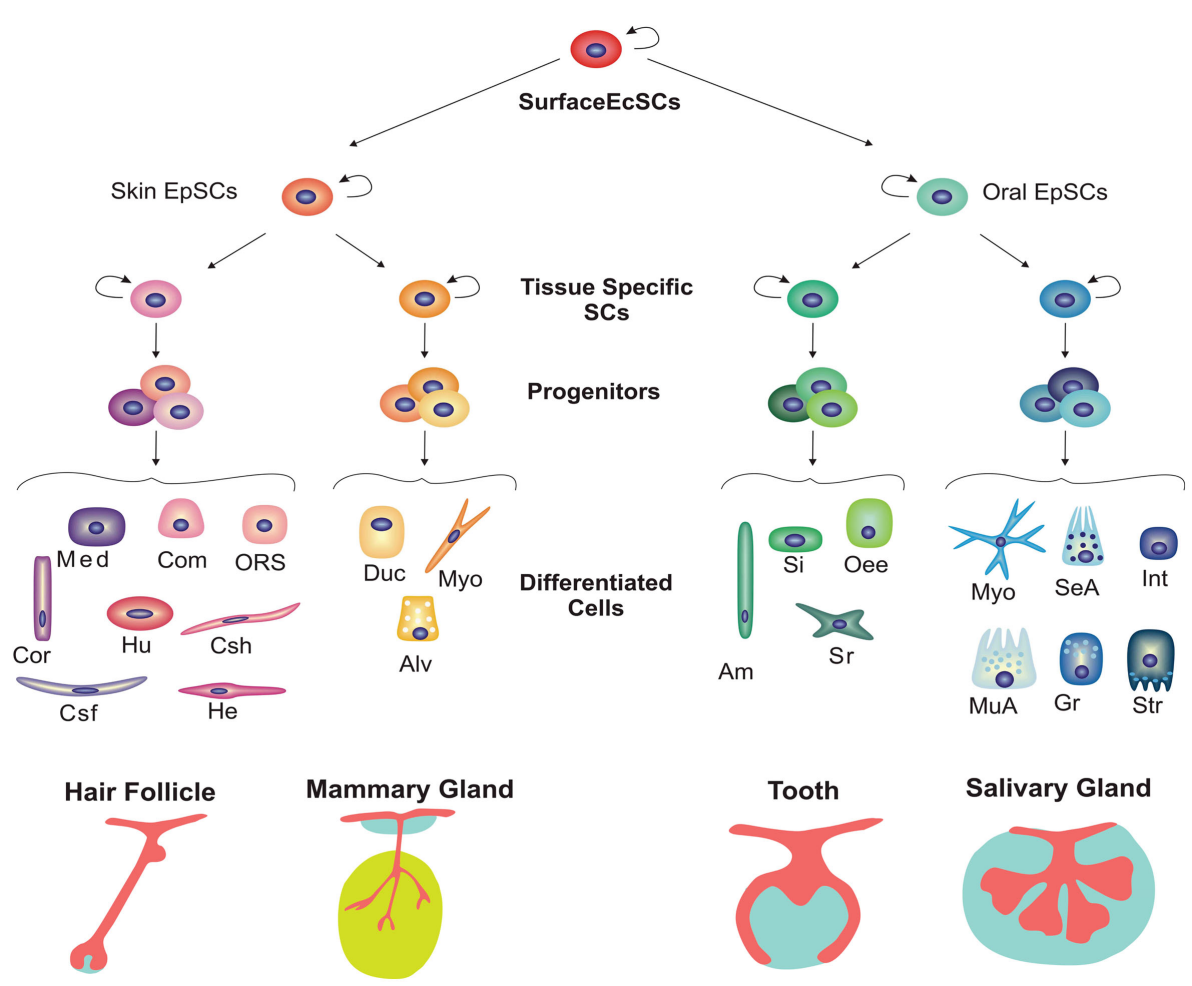

FIGURE 4 | Hierarchical model for stem cell commitment during development of various ectodermal organs. Skin and oral epithelia arise from the surface ectoderm. Putative multipotent stem cells residing in both epithelia can take different fate decisions, giving rise to diverse appendages. During further development of ectodermal organs, more committed tissue-specific stem cells arise from these putative multipotent stem cells in the skin and oral epithelium. Subsequently, tissue-specific stem cells give rise to progenitors or transient amplifying cells that differentiate into functional cell populations. Abbreviations: Alv, alveolar cell; Am, ameloblast; Com, companion layer; Cor, cortex; Csf, cuticle of the hair shalf; Csh, cuticle of the hair sheath; Duc, ductal cell; EcSCs, ectodermal stem cells; EpSCs, epithelial stem cells; Gr, granular duct cell; He, Henle's inner root sheath layer; Hu, Huxley's inner root sheath layer; Int, intercalated duct cell; Med, medulla; Myo, myoepithelial cell; MuA, mucous acinus; Oee, Outer enamel epithelium; ORS, outer root sheath; SeA, serous acinus; Si, stratum intermedium cell; $\mathrm{Sr}$, stellate reticulum cell; Str, striated duct cell.

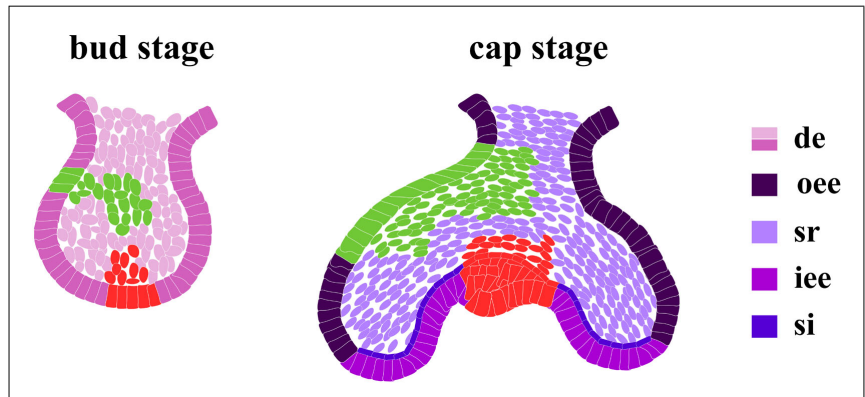

FIGURE 5 | Dil labeling in dental epithelium. At the cap stage, the fate of cells from the dental epithelial bud is already determined. Four different, phenotypically distinct, cell compartments compose the enamel epithelium: outer enamel epithelium (oee); inner enamel epithelium (iee); stellate reticulum (sr); stratum intermedium (si). Two independent Dil labeling experiments (in green and red) traced the fate of cells from different regions in the dental bud epithelium. At a more advanced developmental stage (cap stage) the Dil dye is detected in the same compartments where it was previously injected.

homeostasis and injury-repair (Li and Xie, 2005; Mitsiadis et al., 2007; Barker et al., 2010). Adult stem cells are considered poorly differentiated cells that have self-renewal capacity and can give rise to every cell type in a given tissue (Potten and Loeffler, 1990). Adult stem cells reside in niches that provide them with the proper signals to regulate their function and maintenance according to the tissue requirements ( $\mathrm{Li}$ and Xie, 2005). Deregulation of adult stem cells can result in pathologies such as tumorigenesis or early senescence (Iglesias-Bartolome and Gutkind, 2011).

Ectodermal organs are exposed to a high risk of being damaged and it becomes extremely important that they retain the ability of wound repair and regenerate throughout the organism's lifespan. Similarly to other organs, the injuries suffered after birth by epidermis and oral epithelium, as well as their appendages, are overcome with the help of adult stem cells.

\section{EPITHELIAL ADULT STEM CELLS DURING DEVELOPMENT AND HOMEOSTASIS OF ECTODERMAL APPENDAGES}

In a number of ectodermal organs, epithelial adult stem cells have already been identified. However, in the vast majority, the existence of an adult stem cell pool in the epithelium is suspected although it has not been yet well characterized. Fluorescence-activated cell sorting (FACS) has been widely used to separate the different cell populations residing in a tissue based on their differential expression of several cell surface proteins. Subsequently, their stemness can be assessed either in vitro or in vivo. In vitro, the ability of cells 
to form colonies or spheres containing various lineages has been used to define adult epithelial cells with stem/progenitor properties (Barrandon and Green, 1987; Dontu et al., 2003; Blanpain et al., 2004; Lombaert et al., 2008). Moreover, when disaggregated and replated as single cells, putative stem cells can form new colonies or spheres, showing self-renewal capacity (Barrandon and Green, 1987; Dontu et al., 2003). In vivo, transplantation assays have largely been used to prove that a given cell behaves as a stem cell in hair follicle (Blanpain et al., 2004), tooth (Ohazama et al., 2004a), mammary (Sleeman et al., 2006), and salivary glands (Lombaert et al., 2008). The previously mentioned transplantation into cleared mammary fat pads and the transplantation of dental cells under kidney capsules to prove their ability to develop toothlike structures, are some examples. In salivary gland stem cell biology, ligation-deligation techniques forcing the putative adult stem cell expansion are used. Briefly, closing or ligating the main duct in a salivary gland causes wide organ damage and atrophy. The subsequent duct deligation, triggers a very intense process of gland regeneration which has been extensively studied to identify the putative adult stem cells (Scott et al., 1999; Carpenter et al., 2009).

Although very informative, most of these techniques do not reflect the physiological situation. Indeed, lineage tracing experiments using inducible Cre-lox technology have recently demonstrated that the potential of cells can be different during normal homeostasis and experimental conditions (van Keymeulen et al., 2011). Thus, there is a need to overcome such technical limitations in order to be able to unequivocally define epithelial adult stem cells in ectodermal appendages. Hereby, we provide an overview of the current knowledge on epithelial adult stem cells in some ectodermal appendages such as hair follicles, mammary glands, teeth, and salivary glands during normal development.

Similar to other rapidly renewing organs, quiescent, and active adult stem cells coexist in hair follicles (Li and Clevers, 2010). Cotsarelis and colleagues first proposed the presence of slow cycling and label retaining stem cells. Those cells are residing in the bulge, a small area close to the attachment-site of the erector pili (Cotsarelis et al., 1990). Bulge stem cells express the surface marker CD34 as well as a truncated isoform of Keratin 15, and they have the ability to form colonies in vitro and produce fully functional hair follicle and interfollicular epidermis in vivo (Trempus et al., 2003; Blanpain et al., 2004; Morris et al., 2004). Apart from the slow cycling bulge stem cells, actively proliferating stem cells have been also described. These stem cells express Leucine-rich $G$ proteincoupled receptor 5 (Lgr5) and reside not only in the bulge but also in the hair germ. Lgr5-positive stem cells contribute to the renewal of the lower hair follicle components under physiological conditions, although they can be forced to regenerate the entire hair follicle, the sebaceous gland, and the interfollicular epithelium in transplantation assays (Jaks et al., 2008).

The existence of adult mammary stem cells (MaSCs) is strongly supported by the fact that any portion of the adult mouse mammary epithelium, at any developmental stage, is able to grow and give rise to an entire new ductal system when transplanted into epithelium-free fat pads (DeOme et al., 1959; Smith and Medina, 1988). However, the newly formed mammary epithelium can only be re-transplanted for approximately seven generations, suggesting that MaSCs have a finite life span (Daniel et al., 1968).
Further attempts to characterize MaSCs using FACS revealed that $\mathrm{Cd} 24^{\text {low }} \mathrm{Cd} 49 \mathrm{f}^{\text {high }}$ and $\mathrm{Cd} 24^{\text {low }} \mathrm{Cd} 29^{\text {high }}$ populations are enriched for MaSCs, based on their high regeneration ability when injected into cleared fat pads (Shackleton et al., 2006; Sleeman et al., 2006; Stingl et al., 2006). However, recent data based on lineage tracing experiments suggest that during postnatal mammary gland development, newly generated luminal or myoepithelial cells arise from unipotent progenitors rather than from multipotent adult stem cells (van Keymeulen et al., 2011).

Multiple lines of evidence support the existence of adult stem cells in salivary glands. Radiolabeling experiments have demonstrated the presence of multipotent cells in the intercalated ducts that can contribute to both acinar and ductal cell lineages (Zajicek et al., 1985; Man et al., 2001). More recently, Kishi et al. (2006) used a clonal assay to demonstrate the existence of common progenitors among neonatal rat submandibular gland cells. Damage-induced regeneration assays have served to isolate adult stem cell enriched populations from adult salivary glands based on their c-Kit expression (Lombaert et al., 2008). Moreover, Lombaert and colleagues isolated mouse submandibular gland cells from the duct area and cultured them in vitro in a sphere-forming assay. Thus, when c-Kit-positive cells were purified by FACS and transplanted into an irreversibly damaged mouse submandibular gland they were able to regenerate a functional gland, exhibiting stem cell properties.

Mouse incisors present a source of dental epithelial stem cells that allows them growing continuously throughout the animal's life (Harada et al., 2002). These stem cells reside in the so-called cervical loop present in the labial side of the incisor. The differentiation process occurs along the epithelium from the labial side of the incisor starting from the cervical loop that constitutes the niche for such adult epithelial stem cells. Nevertheless, in mouse molars and human teeth, most of dental epithelium disappears shortly after tooth eruption. The only remaining epithelial cells are the epithelial cell rests of Mallassez (ERM), located in the periodontal ligament tissue. Although their function still remains unclear, sub-cultured ERM have the potential to differentiate into ameloblast-like cells, suggesting that they may be quiescent stem cells (Shinmura et al., 2008).

\section{FLEXIBILITY OF ADULT STEM CELLS DURING REGENERATION}

Adult stem cells have the capacity to give rise to all the tissuespecific progeny required during normal development and tissue homeostasis. However, epithelial adult stem cells retain some flexibility (or plasticity) that provides them the ability to contribute to the formation of different organs during regeneration processes triggered by wound repair or in the previously mentioned recombination assays. For instance, this plasticity has been observed in hair follicle adult stem cells during wound healing of epidermis. Stem cells from the bulge region of hair follicles contribute only to hair follicle progenies during both embryonic development and adult tissue homeostasis but they can also contribute to interfollicular epidermis during wound healing (Levy et al., 2005, 2007; Blanpain, 2010). Similarly, lineage tracing experiments in the mammary gland have recently demonstrated that although during normal postnatal development and homeostasis, cells arise only from committed progenitors, myoepithelial progenitors can be forced to be multipotent in mammary reconstitution assays 
(van Keymeulen et al., 2011). In addition, putative salivary adult stem cells have the ability to give rise to pancreas and liver lineages in vitro (Hisatomi et al., 2004).

Thus, the plasticity of adult stem cells represents an advantage for the potential use of stem cell therapies in regenerative medicine. For instance, due to the lack of dental epithelium in erupted human teeth, obtaining functional enamel-producing ameloblasts from a non-dental source of stem cells constitutes a major challenge in the dental regeneration field. Hence, it has been shown that stem cells derived from other tissues such as bone marrow can contribute to the ameloblasts layer in the in vivo formed tooth after recombining them with dental and mesenchymal cells ( $\mathrm{Hu}$ et al., 2006). Nevertheless, in this case, dental epithelial cells were still needed for an organized tooth reconstitution. Given their common origin, it is conceivable that adult stem cells from other ectodermal-derived tissues may be able to differentiate into dental epithelium. In fact, recombination of postnatal non-dental oral epithelium and embryonic dental mesenchyme gives rise to toothlike structures demonstrating that, similar to hair follicle stem cells, adult oral epithelial stem cells retain a certain plasticity (Nakagawa et al., 2009). Further investigations to gain a better knowledge on the molecular and cellular mechanisms involved in ectodermal organs' development and injury response are still required in order to successfully apply the regenerative therapies to human diseases and tissue damage.

\section{SIGNALING PATHWAYS INVOLVED IN THE REGULATION OF ECTODERMAL STEM CELLS}

Several signaling pathways coordinately regulate the initiation and further development of ectodermal organs. The study of transgenic animals has shed some light on which molecules regulate the processes of specification of the skin or oral ectodermal stem cells into diverse tissue-specific progenitors, as well as the consequent fate choices to give rise to the distinct cell populations that will build up the organ.

\section{BONE MORPHOGENETIC PROTEINS}

Bone morphogenetic proteins (BMPs) are secreted growth factors that belong to the transforming growth factor beta (TGF $\beta$ ) superfamily (Zeng et al., 2010). They were first described by their ability to induce bone formation, but since then, they have been implicated in several processes such as proliferation, apoptosis, tumorigenesis, tissue repair, stem cell maintenance, and epithelial cell fate determination (Wagner et al., 2010). BMP signaling is activated when BMP ligand dimers bind to the receptor complex. Two different types of serine/threonine kinase receptors form the BMP receptor complex: BMP receptor type I (BMPRI) and type II (BMPRII). When the signaling is activated, BMPRII receptors phosphorylate BMPRI receptors, which in turn phosphorylate the cytoplasmic common mediator Smad (co-Smad or Smad4) inducing its binding to the receptor-activated Smads (RSmads). Subsequently, the complex formed by the co-Smad and $\mathrm{R}-\mathrm{Smads}$ is translocated to the nucleus where it binds to the BMP response elements (BRE) in the promoter of BMP target genes, inducing or inhibiting their expression. BMP signaling is regulated at the extracellular level by various molecules that function as BMP antagonists, including Noggin, Chordin, Follistatin and follistatin-related gene product (FLRG), Ventroptin, twisted gastrulation (Twsg1), and the Dan/cerberus family of proteins (Zeng et al., 2010).

During early embryogenesis in vertebrates, BMP4 is considered to be the signal that induces the epidermal vs neural fate of the ectoderm at gastrulation stage (Wilson and Hemmati-Brivanlou, 1995). The levels of BMPs have shown to be important in the fate determination of later epidermal progenitors during embryogenesis. Thus, lowering the levels of BMP signaling by conditional overexpression of Noggin, a BMP antagonist, results in defects in ectodermal organs due to inappropriate fate inductions. For instance, K14-Noggin overexpressing mice show replacement of sweat glands by pilosebaceous units (Plikus et al., 2004) and conversion of the nipple to hair-bearing epithelia (Mayer et al., 2008). Similarly, the blockage of BmpRIb-mediated signaling in chicken embryos leads to the transformation of dorsal scales to feather filaments in the dorsal surface of the foot (Zou and Niswander, 1996). Expression of BMP4 in the early oral epithelium has been shown to be necessary for the induction of tooth formation (Ohazama et al., 2004b) and Bmp7 deficient mice occasionally lack teeth (Zouvelou et al., 2009). Moreover, Cre-mediated loss of epithelial Bmprla results in striking defects in postnatal hair follicle differentiation, as well as in an early arrest of tooth morphogenesis at bud stage (Andl et al., 2004). Thus, BMP signaling plays an essential role in the organ-type specification and development initiation of different organs from ectodermal origin.

\section{NOTCH}

Notch signaling is a highly conserved pathway that involves cell-cell contact. In mammals, 4 receptors have been described (Notch1Notch4) with 5 ligands (Jagged1, Jagged2, Delta-like1, Delta-like3, and Delta-like4). Upon ligand-receptor binding, the extracellular domain of Notch receptors is proteolytically cleaved. Subsequently, the intracellular domain (NICD) is translocated to the nucleus where it binds to the transcription factor CSL (CBF-1 in humans or RBP-J in mice), forming a complex that regulates the transcription of Notch target genes (Figure 6). Notch signaling has diverse and multiple functions on cell differentiation, proliferation, and survival.

The Notch pathway is a master regulator of cell fate determination. In invertebrates, Notch activation directs accurate cell fate choices by restricting differentiation toward alternative fates and permits the self-renewal and survival of multipotent cells (Artavanis-Tsakonas et al., 1999). In vertebrates, diverse cell types express the different Notch receptors and ligands, indicating that Notch signaling may have more complex functions (Figure 7). Notch signaling regulates the switch from basal to suprabasal fate in epidermis (Blanpain et al., 2006) and controls differentiation and homeostasis in hair follicles (Pan et al., 2004). In the mammary gland, Notch activation specifies the luminal cell fate (Yalcin-Ozuysal et al., 2010). In the developing tooth, Notch signaling has been proposed as an important regulator of dental cell type specification in the enamel organ (Mitsiadis and Graf, 2009). Notch 1 is expressed in stratum intermedium, whereas Jagged 2 and Delta-like1 are expressed in the adjacent cell layer of inner enamel epithelium, suggesting that Jagged 2 and Delta-like1 proteins may function as ligands for Notch1 receptor during tooth development 


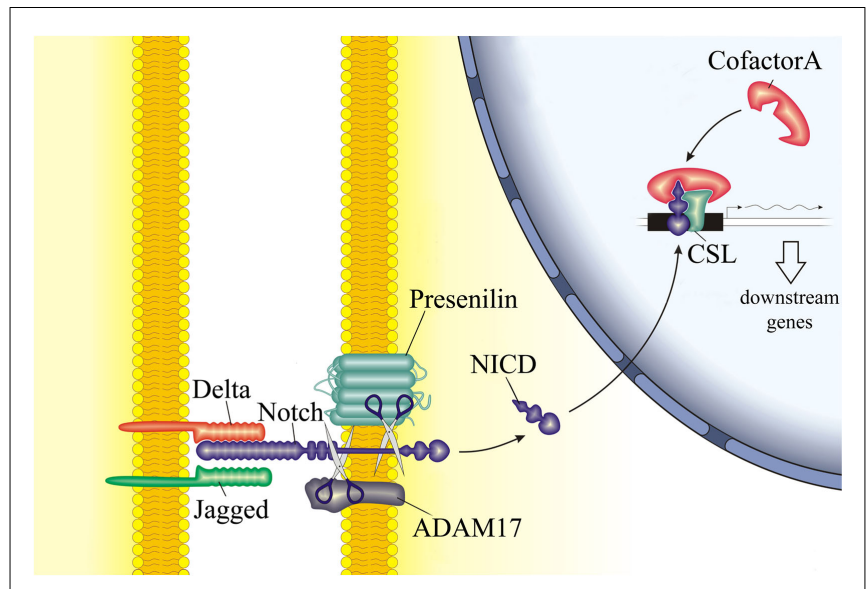

FIGURE 6 | Schematic representation of the Notch signaling pathway. The interaction between Notch receptor on one cell and its ligands on the neighboring cell results in the proteolytic cleavage of Notch receptor and release of its inter-cellular domain (NICD) into the cytoplasm. NICD therefore translocates to the nucleus and interacts with cofactors to regulate the expression of downstream genes.

(Mitsiadis et al., 1995, 1997, 1998, 2010). It is possible that cell fate determination in developing dental epithelium occurs through inhibitory interactions between adjacent epithelial cells. Notch signaling seems to have an essential role in those interactions, influenced by extrinsic signals belonging to other pathways such as fibroblast growth factor (FGF) and BMP signaling pathways (Mitsiadis and Graf, 2009; Mitsiadis et al., 2010).

\section{WINGLESS-TYPE MMTV INTEGRATION SITE (Wnt)}

Wnt ligands are secreted, cysteine-rich proteins that signal through transmembrane receptors belonging to the Frizzled family (Wodarz and Nusse, 1998). Wnt signaling can activate both canonical and non-canonical pathways. The canonical transduction pathway involves the activation of the intracellular protein Disheveled (Dsh). $\beta$-Catenin is consequently stabilized and then translocated to the nucleus where it forms complexes with TCF/LEF transcription factors regulating the expression of target genes.

Wnt signaling has been implicated in the induction of placode formation during early developmental stages of several ectodermal organs (Andl et al., 2002; Chu et al., 2004). Hyper-activation of canonical Wnt signaling in the mouse oral epithelium results in the formation of supernumerary teeth due to a continuous induction of dental epithelium thickening and subsequent tooth development throughout life (Järvinen et al., 2006). Lef-1 is a Wnt target transcription factor that it is also involved in the transduction of canonical Wnt signaling. Deletion of Lef- 1 in mice results in the developmental arrest of ectodermal organs such as mammary gland, hair, and tooth at the bud stage. Lef- 1 overexpression under the Keratin14 (K14) promoter in mice leads to an inappropriate positioning of hair follicles in the skin (Zhou et al., 1995). Moreover, an ectopic formation of tooth-like structures and hair follicles in lip furrow epithelium is apparent in these mice. Interestingly, the expression of $\mathrm{K} 14$, and thus Lef-1, is higher in that region than in the rest of the oral epithelium. Taken together, these data

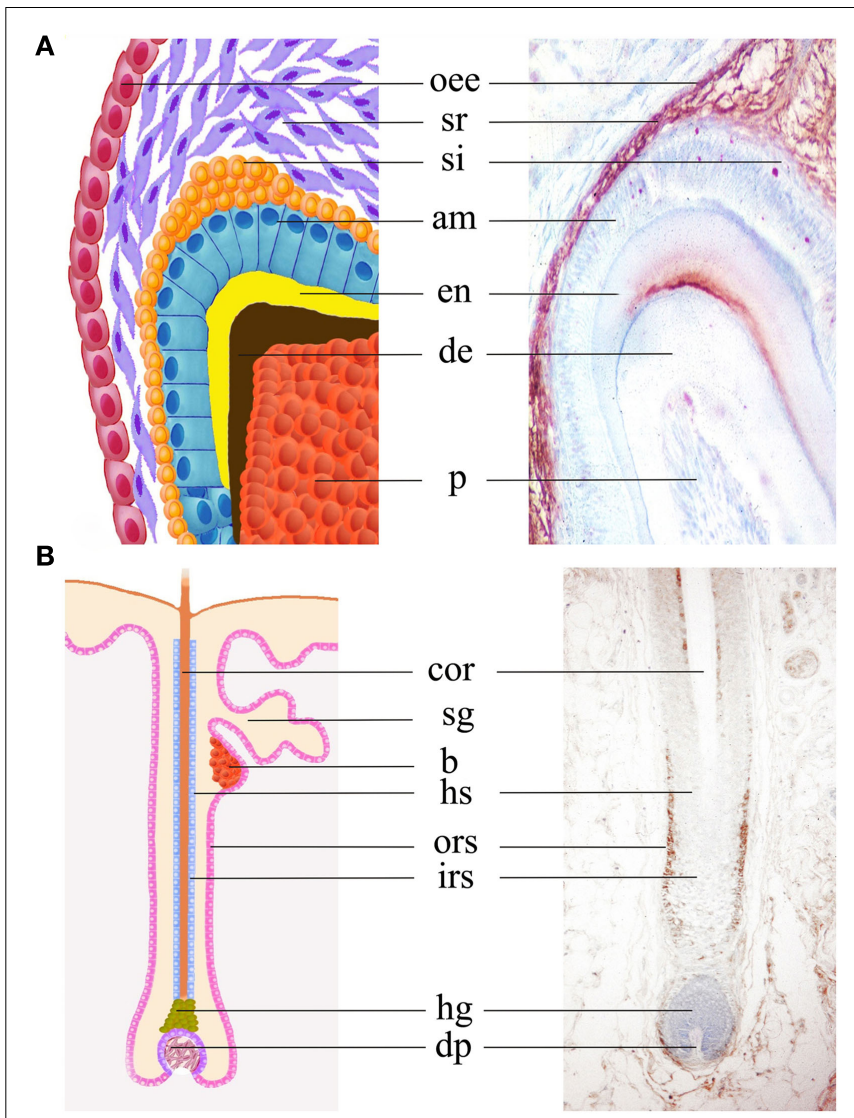

FIGURE 7 | Expression of the Notch2 protein in molar teeth and hair follicles. Immunohistochemical staining (brown color) on sections shows Notch2 expression in stellate reticulum, stratum intermedium, outer enamel epithelium, and dental papilla in postnatal day 6 mouse molars (A). In human hair follicles, Notch2 protein is mainly detected in outer root sheath (B). Abbreviations: am, ameloblast; b, bulge; cor, cortex; de, dentin; $\mathrm{dp}$, derma papilla; en, enamel; irs, inner root sheath; hg, hair germ; hs, hair sheath; oee, outer enamel epithelium; ors, outer root sheath; p, pulp; si, stratum intermedium; sg, salivary gland; sr, stellate reticulum.

suggest that Wnt signaling activation is necessary for the induction of ectodermal appendages during normal development. Furthermore, its activation levels are crucial in regulating ectodermal stem cell fate, forcing them to form appendages in ectopic sites when altered.

\section{FIBROBLAST GROWTH FACTORS}

The FGF family is composed of 22 secreted polypeptides that bind to specific transmembrane tyrosine kinase receptors. To date, four different $F g f r$ genes have been described ( $F g f r 1-F g f r 4$ ) encoding 7 different major proteins (FGFRs 1b, 1c, 2b, 2c, 3b, 3c, and 4) with differing ligand-binding specificity (Itoh and Ornitz, 2011). FGFs are among the main regulators of embryonic development controlling cell proliferation, survival, and differentiation (Szebenyi and Fallon, 1999).

The study of different mouse models in which FGF signaling is altered has demonstrated their involvement in epithelialmesenchymal interactions that characterize the development of ectodermal organs. Abrogation of FGF signaling has been related 
to skin alterations and a defective wound healing capacity (Werner et al., 1994; Ortega et al., 1998). Regarding skin appendages, a number of FGF mutants with hair defects exist (Hébert et al., 1994; Petiot et al., 2003). In addition, FGF8, FGFR1, and FGF10/FGFR2b signaling have been shown to be important during the early stages of mammary gland development (Mailleux et al., 2002; Eblaghie et al., 2004) pointing to FGFs as essential regulators of the specification of the ectodermal stem cells from the ventral skin to form mammary placodes during embryogenesis. FGFs have also been implicated in the development of oral ectoderm-derived organs such as salivary gland and tooth. In the salivary gland, FGF10 is crucial for the initiation and correct patterning of submandibular salivary gland (Ohuchi et al., 2000). During early odontogenesis, FGF signaling determines the position and patterning of teeth (Neubüser et al., 1997). Moreover, FGFs have been described as important regulators of dental epithelial stem cells. In the absence of FGF10, the putative stem cell niche termed cervical loop cannot be maintained and mouse incisors cease their growth (Harada et al., 2002). In conclusion, FGFs function as inductors of ectodermal appendage development and regulators of tissue-specific stem cells residing in the epithelium of different ectodermal-derived organs such as epidermis and teeth.

\section{ECTODYSPLASIN A (EDA)}

EDA is a member of the tumor necrosis factor (TNF) superfamily. Mice carrying mutations in Eda gene present defects in the development of several ectodermal organs such as hair, teeth, and sweat glands (Mikkola et al., 1999). Interestingly, mice overexpressing EDA in developing epidermis present supernumerary teeth and mammary glands. Furthermore, treating embryonic skin with recombinant EDA induces the formation of epithelial placodes (Mustonen et al., 2004).

\section{ECTODERMAL DYSPLASIAS}

Several types of tissue-specific diseases often affect ectodermderived organs. The existence of disorders affecting the development of more than one of these organs suggests that the function of a putative stem cell residing in the embryonic ectoderm may be altered. Ectodermal dysplasias are defined as a highly heterogeneous group of rare heritable disorders characterized by defects in two or more organs of ectodermal origin (Pinheiro and Freire-Maia, 1994). Dysregulation of genes involved in the normal ectodermal organogenesis can lead to ectodermal dysplasia. This is the case of the transcription factor p63, in which mutations in humans can be the cause of various ectodermal dysplasias (Vanbokhoven et al., 2011). Studies on p63 knockout mice have demonstrated that the $p 63$ gene is crucial for the appropriate stratification of skin epidermis and the correct development of several ectodermal appendages (Mills et al., 1999; Yang et al., 1999). Furthermore, other mutations in molecules involved in the EDA signaling pathway cause severe pathologies

\section{REFERENCES}

Andl, T., Ahn, K., Kairo, A., Chu, E. Y., Wine-Lee, L., Reddy, S. T., Croft,

N. J., Cebra-Thomas, J. A., Metzger, D., Chambon, P., Lyons, K.

M., Mishina, Y., Seykora, J. T., and
Crenshaw, E. B. III, Millar, S. E. (2004). Epithelial Bmprla regulates differentiation and proliferation in tial for tooth development. Development 131, 2257-2268. postnatal hair follicles and is essen-

in both humans and mice, such as hypohidrotic ectodermal dysplasia (Itin and Fistarol, 2004). Ectodermal dysplasias are often characterized by the absence of some ectodermal appendages from the embryonic stages. Thus, these disorders are likely the consequence of a failure during the process of stem cell specification that may be caused by either the loss of the potency or the self-renewal and proliferation capacity of early ectodermal stem cells.

\section{CONCLUDING REMARKS}

Ectoderm-derived skin and oral cavity, as well as the highly specialized appendages that arise from them, are in close contact with the environment. Consequently, these organs are exposed to a great risk of being damaged and it becomes extremely important that they retain regeneration capacity throughout the organisms' life. Adult stem/progenitor cells represent the source for the repair of ectoderm-derived organs during tissue homeostasis as well as upon injury conditions. However, this source may be limited due to aging, or due to certain pathological conditions, leading to the inability of stem cells to repair the damaged tissue. Regenerative medicine tries to overcome these limitations.

Major efforts are being made to isolate and characterize putative adult stem cells in different ectodermal organs. However, in most of the cases, only stem cell enriched populations have been isolated, probably including not only putative stem cells but also more differentiated cell types. Several assays have been developed to assess the regeneration potential of putative stem cells in order to define their stemness. It is essential to bear in mind that those assays may not reflect the normal developmental process but instead they reproduce an injury-repair situation where stem cells may be forced to be more plastic. Thus, in situ lineage tracing experiments provide valuable information concerning cell fate determination under more physiological conditions.

Data obtained from the study of diverse knockout and transgenic mice have improved the knowledge on the regulation of stem cell fate determination at different developmental stages of ectodermal appendages. Signaling molecules belonging to different pathways have been shown to have important roles in such processes. Nevertheless, the tightly controlled interaction in time and space between these signaling pathways still remains unclear. Thus, a better understanding of the molecular mechanisms underlying cell fate choices during normal development and homeostasis of ectodermal-derived organs is mandatory in order to successfully make use of stem cell biology-based therapies in regenerative medicine.

\section{ACKNOWLEDGMENTS}

This work was supported by the 3100A0-118332 SNSF grant (Thimios A. Mitsiadis, Zoraide Granchi) and funds from the University of Zurich (Lucía Jiménez-Rojo, Daniel Graf, Thimios A. Mitsiadis).

Andl, T., Reddy, S. T., Gaddapara, T., and Millar, S. E. (2002). WNT signals are required for the initiation of hair follicle development. Dev. Cell 2, 643-653.
Artavanis-Tsakonas, S., Rand, M. D. and Lake, R. J. (1999). Notch signaling: cell fate control and signal integration in development. Science 284, 770-776. 
Barker, N., Bartfeld, S., and Clevers, H. (2010). Tissue-resident adult stem cell populations of rapidly selfrenewing organs. Cell Stem Cell 7, 656-670.

Barrandon, Y., and Green, H. (1987). Three clonal types of keratinocyte with different capacities for multiplication. Proc. Natl. Acad. Sci. U.S.A. 84, 2302-2306.

Blanpain, C. (2010). Stem cells: skin regeneration and repair. Nature 464 , 686-687.

Blanpain, C., Lowry, W. E., Geoghegan, A., Polak, L., and Fuchs, E. (2004). Self-renewal, multipotency, and the existence of two cell populations within an epithelial stem cell niche. Cell 118, 635-648.

Blanpain, C., Lowry, W. E., Pasolli, H. A., and Fuchs, E. (2006). Canonical notch signaling functions as a commitment switch in the epidermal lineage. Genes Dev. 20, 3022-3035.

Booth, B. W., Mack, D. L., AndroutsellisTheotokis, A., McKay, R. D. G., Boulanger, C. A., and Smith, G. H. (2008). The mammary microenvironment alters the differentiation repertoire of neural stem cells. Proc. Natl. Acad. Sci. U.S.A. 105, 14891-14896.

Boulanger, C. A., Bruno, R. D., Rosu-Myles, M., and Smith, G. H. (2012). The mouse mammary microenvironment redirects mesoderm-derived bone marrow cells to a mammary epithelial progenitor cell fate. Stem cells Dev. 21, 948-954.

Boulanger, C. A., Mack, D. L., Booth, B. W., and Smith, G. H. (2007). Interaction with the mammary microenvironment redirects spermatogenic cell fate in vivo. Proc. Natl. Acad. Sci. U.S.A. 104, 3871-3876.

Carlson, B. (2004). Human Embryology and Developmental Biology, 3rd Edn. Mosby.

Carpenter, G. H., Khosravani, N., Ekström, J., Osailan, S. M., Paterson, K. P., and Proctor, G. B. (2009). Altered plasticity of the parasympathetic innervation in the recovering rat submandibular gland following extensive atrophy. Exp. Physiol. 94, 213-219.

Chu, E. Y., Hens, J., Andl, T., Kairo, A., Yamaguchi, T. P., Brisken, C., Glick, A., Wysolmerski, J. J., and Millar, S. E. (2004). Canonical WNT signaling promotes mammary placode development and is essential for initiation of mammary gland morphogenesis. Development 131, 4819-4829.

Cotsarelis, G., Sun, T. T., and Lavker, R. M. (1990). Label-retaining cells reside in the bulge area of pilosebaceous unit: implications for follicular stem cells, hair cycle, and skin carcinogenesis. Cell 61, 1329-1337.

Cunha, G. R., Young, P., Christov, K., Guzman, R., Nandi, S., Talamantes, F., and Thordarson, G. (1995). Mammary phenotypic expression induced in epidermal cells by embryonic mammary mesenchyme. Acta Anat. (Basel) 152, 195-204.

Daniel, C. W., De Ome, K. B., Young, J. T., Blair, P. B., and Faulkin, L. J. (1968). The in vivo life span of normal and preneoplastic mouse mammary glands: a serial transplantation study. Proc. Natl. Acad. Sci. U.S.A.61, 53-60.

DeOme, K. B., Faulkin, L. J., Bern, H. A., and Blair, P. B. (1959). Development of mammary tumors from hyperplastic alveolar nodules transplanted into gland-free mammary fat pads of female C3H mice. Cancer Res. 19 , 515-520.

Dhouailly, D., Rogers, G. E., and Sengel, P. (1978). The specification of feather and scale protein synthesis in epidermal-dermal recombinations. Dev. Biol. 65, 58-68.

Dontu, G., Abdallah, W. M., Foley, J. M., Jackson, K. W., Clarke, M. F., Kawamura, M. J., and Wicha, M. S. (2003). In vitro propagation and transcriptional profiling of human mammary stem/progenitor cells. Genes Dev. 17, 1253-1270.

Eblaghie, M. C., Song, S.-J., Kim, J.Y., Akita, K., Tickle, C., and Jung, H.-S. (2004). Interactions between FGF and Wnt signals and Tbx 3 gene expression in mammary gland initiation in mouse embryos. J. Anat 205, 1-13.

Harada, H., Toyono, T., Toyoshima, K., Yamasaki, M., Itoh, N., Kato, S., Sekine, K., and Ohuchi, H. (2002). FGF10 maintains stem cell compartment in developing mouse incisors. Development 129, 1533-1541.

Hébert, J. M., Rosenquist, T., Götz, J., and Martin, G. R. (1994). FGF5 as a regulator of the hair growth cycle: evidence from targeted and spontaneous mutations. Cell 78, 1017-1025.

Hisatomi, Y., Okumura, K., Nakamura, K., Matsumoto, S., Satoh, A., Nagano, K., Yamamoto, T., and Endo, F. (2004). Flow cytometric isolation of endodermal progenitors from mouse salivary gland differentiate into hepatic and pancreatic lineages. Hepatology 39, 667-675.

$\mathrm{Hu}$, B., Unda, F., Bopp-Kuchler, S., Jimenez, L., Wang, X. J., Haïkel, Y., Wang, S. L., and Lesot, H. (2006).
Bone marrow cells can give rise to ameloblast-like cells. J. Dent. Res. 85 , 416-421.

Iglesias-Bartolome, R., and Gutkind, J. S. (2011). Signaling circuitries controlling stem cell fate: to be or not to be. Curr. Opin. Cell Biol. 23, 716-723.

Itin, P. H., and Fistarol, S. K. (2004). Ectodermal dysplasias. Am. J. Med Genet. C Semin. Med. Genet. 131C, 45-51.

Itoh, N., and Ornitz, D. M. (2011) Fibroblast growth factors: from molecular evolution to roles in development, metabolism and disease. $J$. Biochem. 149, 121-130.

Jaks, V., Barker, N., Kasper, M., van Es, J. H., Snippert, H. J., Clevers, H., and Toftgård, R. (2008). Lgr5 marks cycling, yet long-lived, hair follicle stem cells. Nat. Genet. 40, 1291-1299.

Järvinen, E., Salazar-Ciudad, I., Birchmeier, W., Taketo, M. M., Jernvall, J., and Thesleff, I. (2006). Continuous tooth generation in mouse is induced by activated epithelial Wnt/beta-catenin signaling. Proc. Natl. Acad. Sci. U.S.A. 103 18627-18632.

Kishi, T., Takao, T., Fujita, K., and Taniguchi, H. (2006). Clonal proliferation of multipotent stem/progenitor cells in the neonatal and adult salivary glands. Biochem. Biophys. Res. Commun. $340,544-552$.

Kollar, E. J., and Baird, G. R. (1969). The influence of the dental papilla on the development of tooth shape in embryonic mouse tooth germs. J. Embryol. Exp. Morphol. 21, 131-148.

Kratochwil, K. (1969). Organ specificity in mesenchymal induction demonstrated in the embryonic development of the mammary gland of the mouse. Dev. Biol. 20, 46-71.

Kusakabe, M., Sakakura, T., Sano, M., and Nishizuka, Y. (1985) A pituitary-salivary mixed gland induced by tissue recombination of embryonic pituitary epithelium and embryonic submandibular gland mesenchyme in mice. Dev. Biol. 110 382-391.

Levy, V., Lindon, C., Harfe, B. D. and Morgan, B. A. (2005). Distinct stem cell populations regenerate the follicle and interfollicular epidermis. Dev. Cell 9, 855-861.

Levy, V., Lindon, C., Zheng, Y., Harfe, B. D., and Morgan, B. A. (2007). Epidermal stem cells arise from the hair follicle after wounding. FASEB J. 21 , 1358-1366.
Li, L., and Clevers, H. (2010). Coexistence of quiescent and active adult stem cells in mammals. Science 327 , 542-545.

Li, L., and Xie, T. (2005). Stem cell niche: structure and function. Annu. Rev. Cell Dev. Biol. 21, 605-631.

Lombaert, I. M. A., Brunsting, J. F., Wierenga, P. K., Faber, H., Stokman, M. A., Kok, T., Visser, W. H., Kampinga, H. H., de Haan, G., and Coppes, R. P. (2008). Rescue of salivary gland function after stem cell transplantation in irradiated glands. PLoS ONE 3, e2063. doi:10.1371/journal.pone.0002063

Mailleux, A. A., Spencer-Dene, B., Dillon, C., Ndiaye, D., Savona-Baron, C., Itoh, N., Kato, S., Dickson, C., Thiery, J. P., and Bellusci, S. (2002). Role of FGF10/FGFR2b signaling during mammary gland development in the mouse embryo. Development 129, 53-60.

Man, Y. G., Ball, W. D., Marchetti, L., and Hand, A. R. (2001). Contributions of intercalated duct cells to the normal parenchyma of submandibular glands of adult rats. Anat. Rec. 263, 202-214.

Mayer, J. A., Foley, J., De La Cruz, D., Chuong, C.-M., and Widelitz, R. (2008). Conversion of the nipple to hair-bearing epithelia by lowering bone morphogenetic protein pathway activity at the dermal-epidermal interface. Am. J. Pathol. 173, 1339-1348.

Mikkola, M. L., Pispa, J., Pekkanen, M., Paulin, L., Nieminen, P., Kere, J., and Thesleff, I. (1999). Ectodysplasin, a protein required for epithelial morphogenesis, is a novel TNF homologue and promotes cellmatrix adhesion. Mech. Dev. 88, 133-146.

Mills, A. A., Zheng, B., Wang, X. J., Vogel, H., Roop, D. R., and Bradley, A. (1999). p63 is a p53 homologue required for limb and epidermal morphogenesis. Nature 398, 708-713.

Mina, M., and Kollar, E. J. (1987) The induction of odontogenesis in non-dental mesenchyme combined with early murine mandibular arch epithelium. Arch. Oral Biol. 32, 123-127.

Mitsiadis, T. A., Barrandon, O., Rochat, A., Barrandon, Y., and De Bari, C. (2007). Stem cell niches in mammals. Exp. Cell Res. 313, 3377-3385.

Mitsiadis, T. A., Caton, J., and Cobourne, M. (2006). Wakingup the sleeping beauty: recovery of the ancestral bird odontogenic program. J. Exp. Zool. B Mol. Dev. Evol. 306, 227-233. 
Mitsiadis, T. A., Chéraud, Y., Sharpe, P., and Fontaine-Pérus, J. (2003). Development of teeth in chick embryos after mouse neural crest transplantations. Proc. Natl. Acad. Sci. U.S.A. 100, 6541-6545.

Mitsiadis, T. A., and Graf, D. (2009). Cell fate determination during tooth development and regeneration. Birth Defects Res. C Embryo Today 87, 199-211.

Mitsiadis, T. A., Graf, D., Luder, H., Gridley, T., and Bluteau, G. (2010). BMPs and FGFs target Notch signalling via jagged 2 to regulate tooth morphogenesis and cytodifferentiation. Development 137, 3025-3035.

Mitsiadis, T. A., Henrique, D., Thesleff, I., and Lendahl, U. (1997). Mouse Serrate-1 (Jagged-1): expression in the developing tooth is regulated by epithelial-mesenchymal interactions and fibroblast growth factor-4. Development 124, 1473-1483.

Mitsiadis, T. A., Hirsinger, E., Lendahl, U., and Goridis, C. (1998). Deltanotch signaling in odontogenesis: correlation with cytodifferentiation and evidence for feedback regulation. Dev. Biol. 204, 420-431.

Mitsiadis, T. A., Lardelli, M., Lendahl, U., and Thesleff, I. (1995). Expression of Notch 1, 2 and 3 is regulated by epithelial-mesenchymal interactions and retinoic acid in the developing mouse tooth and associated with determination of ameloblast cell fate. J. Cell Biol. 130, 407-418.

Mitsiadis, T. A., Tucker, A. S., De Bari, C., Cobourne, M. T., and Rice, D. P. C. (2008). A regulatory relationship between Tbxl and FGF signaling during tooth morphogenesis and ameloblast lineage determination. Dev. Biol. 320, 39-48.

Morris, R. J., Liu, Y., Marles, L., Yang, Z., Trempus, C., Li, S., Lin, J. S., Sawicki, J. A., and Cotsarelis, G. (2004). Capturing and profiling adult hair follicle stem cells. Nat. Biotechnol.22, 411-417.

Mustonen, T., Ilmonen, M., Pummila, M., Kangas, A. T., Laurikkala, J., Jaatinen, R., Pispa, J., Gaide, O., Schneider, P., Thesleff, I., and Mikkola, M. L. (2004). Ectodysplasin A1 promotes placodal cell fate during early morphogenesis of ectodermal appendages. Development 131, 4907-4019.

Nakagawa, E., Itoh, T., Yoshie, H., and Satokata, I. (2009). Odontogenic potential of post-natal oral mucosal epithelium. J. Dent. Res. 88, 219-223.

Neubüser, A., Peters, H., Balling, R., and Martin, G. R. (1997). Antagonistic interactions between FGF and BMP signaling pathways: a mechanism for positioning the sites of tooth formation. Cell 90, 247-255.

Niemann, C., and Watt, F. M. (2002). Designer skin: lineage commitment in postnatal epidermis. Trends Cell Biol. 12, 185-192.

Ohazama, A., Modino, S. A. C., Miletich, I., and Sharpe, P. T. (2004a). Stem-cell-based tissue engineering of murine teeth. J. Dent. Res. 83, 518-522.

Ohazama, A., Tucker, A., and Sharpe, P. T. (2004b). Organized tooth-specific cellular differentiation stimulated by BMP4. J. Dental Res. 84, 603-606.

Ohuchi, H., Hori, Y., Yamasaki, M., Harada, H., Sekine, K., Kato, S., and Itoh, N. (2000). FGF10 acts as a major ligand for FGF receptor 2 IIIb in mouse multi-organ development. Biochem. Biophys. Res. Commun. 277, 643-649.

Ortega, S., Ittmann, M., Tsang, S. H., Ehrlich, M., and Basilico, C. (1998). Neuronal defects and delayed wound healing in mice lacking fibroblast growth factor 2. Proc. Natl. Acad. Sci. U.S.A. 95, 5672-5677.

Pan, Y., Lin, M.-H., Tian, X., Cheng, H.T., Gridley, T., Shen, J., and Kopan, R. (2004). gamma-secretase functions through Notch signaling to maintain skin appendages but is not required for their patterning or initial morphogenesis. Dev. Cell 7, 731-743.

Petiot, A., Conti, F. J. A., Grose, R., Revest, J.-M., Hodivala-Dilke, K. M., and Dickson, C. (2003). A crucial role for Fgfr2-IIIb signalling in epidermal development and hair follicle patterning. Development 130, 5493-5501.

Pinheiro, M., and Freire-Maia, N. (1994). Ectodermal dysplasias: a clinical classification and a causal review. Am. J. Med. Genet. 53, 153-162.

Pispa, J., and Thesleff, I. (2003). Mechanisms of ectodermal organogenesis. Dev. Biol. 262, 195-205.

Plikus, M., Wang, W. P., Liu, J., Wang, X., Jiang, T.-X., and Chuong, C.-M. (2004). Morpho-regulation of ectodermal organs: integument pathology and phenotypic variations in K14-Noggin engineered mice through modulation of bone morphogenic protein pathway. Am. J. Pathol. 164, 1099-1114.

Potten, C. S., and Loeffler, M. (1990). Stem cells: attributes, cycles, spirals, pitfalls and uncertainties. Lessons for and from the crypt. Development 110, 1001-1020.

Sakakura, T., Kusano, I., Kusakabe, M., Inaguma, Y., and Nishizuka, Y. (1987). Biology of mammary fat pad in fetal mouse: capacity to support development of various fetal epithelia in vivo. Development 100, 421-430.

Sakakura, T., Nishizuka, Y., and Dawe, C. J. (1976). Mesenchymedependent morphogenesis and epithelium-specific cytodifferentiation in mouse mammary gland. Science 194, 1439-1441.

Scott, J., Liu, P., and Smith, P. M. (1999). Morphological and functional characteristics of acinar atrophy and recovery in the duct-ligated parotid gland of the rat. J. Dent. Res. 78 1711-1719.

Sengel, P. (1990). Pattern formation in skin development. Int. J. Dev. Biol. 34, 33-50.

Shackleton, M., Vaillant, F., Simpson, K. J., Stingl, J., Smyth, G. K., AsselinLabat, M.-L., Wu, L., Lindeman, G. J., and Visvader, J. E. (2006). Generation of a functional mammary gland from a single stem cell. Nature 439, 84-88.

Shinmura, Y., Tsuchiya, S., Hata, K.-I., and Honda, M. J. (2008). Quiescent epithelial cell rests of Malassez can differentiate into ameloblast-like cells. J. Cell. Physiol. 217, 728-738.

Sleeman, K. E., Kendrick, H., Ashworth, A., Isacke, C. M., and Smalley, M. J. (2006). CD24 staining of mouse mammary gland cells defines luminal epithelial, myoepithelial/basal and non-epithelial cells. Breast Cancer Res. 8, R7.

Smith, G., and Medina, D. (1988). A morphologically distinct candidate for an epithelial stem cell in mouse mammary gland. J. Cell Sci. 90, 173-183.

Stingl, J., Eirew, P., Ricketson, I., Shackleton, M., Vaillant, F., Choi, D., Li, H. I., and Eaves, C. J. (2006). Purification and unique properties of mammary epithelial stem cells. Nature 439, 993-997.

Szebenyi, G., and Fallon, J. F. (1999). Fibroblast growth factors as multifunctional signaling factors. Int. Rev. Cytol. 185, 45-106.

Trempus, C. S., Morris, R. J., Bortner, C. D., Cotsarelis, G., Faircloth, R. S., Reece, J. M., and Tennant, R. W. (2003). Enrichment for living murine keratinocytes from the hair follicle bulge with the cell surface marker CD34. J. Invest. Dermatol. 120, 501-511.

Tucker, A. S. (2007). Salivary gland development. Semin. Cell Dev. Biol. 18, 237-244.

van Keymeulen, A., Rocha, A. S., Ousset, M., Beck, B., Bouvencourt, G., Rock, J., Sharma, N., Dekoninck, S., and Blanpain, C. (2011). Distinct stem cells contribute to mammary gland development and maintenance. Nature 479, 189-193.

Vanbokhoven, H., Melino, G., Candi, E., and Declercq, W. (2011). p63, a story of mice and men. J. Invest. Dermatol. 131, 1196-1207.

Wagner, D. O., Sieber, C., Bhushan, R., Börgermann, J. H., Graf, D., and Knaus, P. (2010). BMPs: from bone to body morphogenetic proteins. Sci. signaling 3 , mrl.

Werner, S., Smola, H., Liao, X., Longaker, M. T., Krieg, T., Hofschneider, P. H. and Williams, L. T. (1994). The function of KGF in morphogenesis of epithelium and reepithelialization of wounds. Science 266, 819-822.

Wilson, P. A., and Hemmati-Brivanlou, A. (1995). Induction of epidermis and inhibition of neural fate by Bmp-4. Nature 376, 331-333.

Wodarz, A., and Nusse, R. (1998). Mechanisms of Wnt signaling in development. Annu. Rev. Cell Dev. Biol. 14, 59-88.

Woodward, W. A., Chen, M. S., Behbod, F., and Rosen, J. M. (2005). On mammary stem cells. J. Cell. Sci. 118, 3585-3594.

Yalcin-Ozuysal, O., Fiche, M., Guitierrez, M., Wagner, K.-U., Raffoul, W., and Brisken, C. (2010). Antagonistic roles of Notch and p63 in controlling mammary epithelial cell fates. Cell Death Differ. 17, 1600-1612.

Yang, A., Schweitzer, R., Sun, D., Kaghad, M., Walker, N., Bronson, R. T., Tabin, C., Sharpe, A., Caput, D., Crum, C., and McKeon, F. (1999). p63 is essential for regenerative proliferation in limb, craniofacial and epithelial development. Nature 398, 714-718.

Zajicek, G., Yagil, C., and Michaeli, Y. (1985). The streaming submandibular gland. Anat. Rec. 213, 150-158.

Zeng, S., Chen, J., and Shen, H. (2010). Controlling of bone morphogenetic protein signaling. Cell. Signal. 22, 888-893.

Zhou, P., Byrne, C., Jacobs, J., and Fuchs, E. (1995). Lymphoid enhancer factor 1 directs hair follicle patterning and epithelial cell fate. Genes Dev. 9, 700-713.

Zou, H., and Niswander, L. (1996). Requirement for BMP signaling in interdigital apoptosis and scale formation. Science 272, 738-741.

Zouvelou, V., Luder, H.-U., Mitsiadis, T. A., and Graf, D. (2009). Deletion of BMP7 affects the development of bones, teeth, and other ectodermal appendages of the orofacial complex. J. Exp. Zool. B Mol. Dev. Evol. 312B, 361-374.

Conflict of Interest Statement: The authors declare that the research was 
conducted in the absence of any commercial or financial relationships that could be construed as a potential conflict of interest.

Received: 01 March 2012; paper pending published: 19 March 2012; accepted:
03 April 2012; published online: 25 April 2012.

Citation: Jiménez-Rojo L, Granchi Z, Graf D and Mitsiadis TA (2012) Stem cell fate determination during development and regeneration of ectodermal organs. Front. Physio.
3:107.

00107

doi:

10.3389/fphys.2012.

This article was submitted to Frontiers in Craniofacial Biology, a specialty of Frontiers in Physiology.

Copyright (c) 2012 Jiménez-Rojo, Granchi, Graf and Mitsiadis. This is an open-access article distributed under the terms of the Creative Common Attribution Non Commercial License, which permits non-commercial use, distribution, and reproduction in other forums, provided the original authors and source are credited. 